CTP-TAMU-18/97

LPTENS-97/17

hep-th/9704186

\title{
Supersymmetry Without Supersymmetry
}

\author{
M. J. Duff ${ }^{\dagger 1}$, H. Lü ${ }^{\ddagger}$ and C. N. Pope ${ }^{\dagger 2}$ \\ ${ }^{\dagger}$ Center for Theoretical Physics \\ Texas A $3 M$ University, College Station, Texas 77843 \\ ${ }^{\ddagger}$ Laboratoire de Physique Théorique de l'École Normale Supérieure ${ }^{3}$ \\ 24 Rue Lhomond - 75231 Paris CEDEX 05
}

\begin{abstract}
$\underline{\text { ABSTRACT }}$
We present four-dimensional $M$-theory vacua with $N>0$ supersymmetry which, from the perspective of perturbative Type $I I A$ string theory, have $N=0$. Such vacua can appear when the compactifying 7 -manifold is a $U(1)$ fibration. The missing superpartners are Dirichlet 0-branes. Someone unable to detect Ramond-Ramond charge would thus conclude that these worlds have no unbroken supersymmetry. In particular, the gravitinos (and also some of the gauge bosons) are 0-branes not seen in perturbation theory but which curiously remain massless however weak the string coupling.
\end{abstract}

\footnotetext{
${ }^{1}$ Research supported in part by NSF Grant PHY-9411543.

${ }^{2}$ Research supported in part by DOE Grant DE-FG05-91-ER40633.

${ }^{3}$ Unité Propre du Centre National de la Recherche Scientifique, associée à l'École Normale Supérieure et à l'Université de Paris-Sud.
} 


\section{Introduction}

Both perturbative and non-perturbative effects of ten-dimensional superstring theory have now been subsumed by eleven-dimensional $M$-theory [1, 2, 3, 4, 5, 6, 7, 8, 9, 10, 11, 12, 13]. In particular, the $D=10$ Type $I I A$ superstring emerges from $M$-theory compactified on $S^{1}$ [1, 4, 5]. In this picture, the resulting Kaluza-Klein modes are Dirichlet 0-branes [14] with masses proportional to $1 / \lambda$ in the string metric, where $\lambda$ is the string coupling constant. They are thus non-perturbative from the Type $I I A$ perspective. This may also be seen from the fact that perturbative string states carry no Ramond-Ramond $U(1)$ charge whereas the massive Kaluza-Klein modes are necessarily charged under this $U(1)$. $M$ theory, on the other hand, draws no distinction between perturbative and non-perturbative states. An interesting question, therefore, is whether there is any difference in the status of supersymmetry when viewed either from the perturbative Type IIA string or from the vantage point of non-perturbative $M$-theory. In this paper we present four-dimensional $M$-theory vacua with $N>0$ supersymmetry which, from the perspective of perturbative Type $I I A$ string theory, have $N=0$. Such vacua can emerge whenever the compactifying 7-manifold is a $U(1)$ bundle over a 6-manifold. The missing superpartners are Dirichlet 0branes. Someone unable to detect Ramond-Ramond charge would thus conclude that these worlds have no unbroken supersymmetry. In particular, the gravitinos (and also some of the gauge bosons) are 0-branes not seen in perturbation theory but which curiously remain massless however weak the string coupling.

The simplest example of this phenomenon is provided by the maximally-symmetric $S^{7}$ compactification [15] of $D=11$ supergravity [16] which is presumably also an acceptable compactification of $M$-theory. Actually, this is not immediately obvious. In the literature one encounters two attitudes to $M$-theory which from the point of view of perturbative string theory may be called revolutionary and counter-revolutionary. The revolutionary will say that having decided that the fundamental theory is eleven-dimensional $M$-theory whose low energy limit is $D=11$ supergravity, then any vacuum of $D=11$ supergravity is an acceptable vacuum, whether or not it is a vacuum of Type $I I A$ supergravity ?. (K3 17 provides a nice example of a $D=7$ vacuum that follows from $D=11$ but not from Type II A supergravity.) The counter-revolutionary, on the other hand will argue that $M$-theory is nothing more than the strong coupling limit of the Type $I I A$ string and hence the only

\footnotetext{
${ }^{1}$ We are assuming throughout that solutions of Type $I I A$ supergravity may be elevated to solutions of the full Type II A string.
} 
acceptable vacua are those that are also solutions of Type $I I A$ supergravity 2 . If true, one might be tempted to conclude that the compactifying 7 -manifold $\mathcal{M}_{7}$ must necessarily be of the form $\mathcal{M}_{6} \times S^{1}$. See, for example, [18]. However, this turns out to be too strong a requirement even for the conservative. As we shall see, we can satisfy both camps if $\mathcal{M}_{7}$ is a $U(1)$ fibration ${ }^{3}$. Fortunately, $S^{7}$ may be regarded as a $U(1)$ bundle over $C P^{3}$ and is in fact but one example of a whole class of compactifications of $D=11$ supergravity corresponding to Hopf fibrations which therefore also admit the interpretation as vacua of the Type $I I A$ theory [19].

Another reason for trusting that $A d S_{4} \times S^{7}$ is an acceptable vacuum of $M$-theory is that it is simply the $M$-theory membrane solution [20] in the limiting case as we approach the horizon [21, 22].

In [1] the worldsheet action of the $D=10$ Type $I I A$ superstring [23] was derived from the worldvolume action of the $D=11$ supermembrane [24] by identifying the third worldvolume coordinate with the eleventh spacetime coordinate. The same procedure continues to apply if this eleventh coordinate corresponds to a $U(1)$ fiber instead of a circle. Now, however, the membrane worldvolume would itself correspond to a $U(1)$ bundle over the two dimensional worldsheet of the string, rather than a direct product.

We complete this introduction with a caveat: when discussing the four-dimensional Type IIA string spectrum we shall not be interested in magnetically charged states so we shall use the words "perturbative" or "non-perturbative" to mean with or without the inclusion of electrically charged Dirichlet 0-branes. These four-dimensional 0-branes have their ten-dimensional origin in the massive Kaluza-Klein spectrum which is absent in the perturbative Type $I I A$ string. The remaining states in the spectrum have their origin in the massless modes in $D=10$. However, even this sector will display certain non-perturbative features peculiar to the $A d S_{4} \times \mathcal{M}_{6}$ backgrounds. We shall return to this issue in section 6 .

\section{$2 \quad U(1)$ fibrations}

It has long been known that Type $I I A$ supergravity can be obtained by the dimensional reduction of eleven-dimensional supergravity on a circle [25, 26]. In particular, this means that any solution of eleven-dimensional supergravity of the form $\mathcal{M}_{10} \times S^{1}$ can be reinterpreted as a solution of the Type $I I A$ theory. In fact the class of eleven-dimensional

\footnotetext{
${ }^{2}$ Similar remarks apply, mutatis mutandis, to the heterotic string and $M$-theory on $S^{1} / Z_{2}$ [9].

${ }^{3}$ This may come as no surprise to $F$-theorists.
} 
solutions that can be re-interpreted as solutions of the Type IIA theory is much wider than these. To be precise, it is not necessary that the eleven-dimensional solution have a direct-product structure, and in fact any solution that has the form of a $U(1)$ bundle over some ten-dimensional base manifold also admits a ten-dimensional interpretation [19]. For example, this includes the case of the $A d S_{4} \times S^{7}$ solution of eleven-dimensional supergravity, since $S^{7}$ can be written as a $U(1)$ bundle over $C P^{3}$. In configurations of this kind where the bundle is non-trivial, the Kaluza-Klein vector potential arising from the reduction from $D=11$ to $D=10$ has a topologically non-trivial form, analogous to the potential for a magnetic monopole. Note, by the way, that there is no circle which cannot be shrunk to a point in $S^{7}$, in contrast to the direct product $C P^{3} \times S^{1}$. In this section, we shall review the structure of such bundle solutions from the eleven and ten-dimensional viewpoints; further details may be found in [19].

We may begin by considering the bosonic sector of eleven-dimensional supergravity,

$$
\hat{\mathcal{L}}=\hat{e} \hat{R}-\frac{1}{48} \hat{e} \hat{F}_{4}^{2}-\frac{1}{6} *\left(d \hat{A}_{3} \wedge d \hat{A}_{3} \wedge \hat{A}_{3}\right) .
$$

After dimensional reduction to $D=10$ according to the Kaluza-Klein prescription

$$
\begin{aligned}
d \hat{s}^{2} & =e^{-\frac{2}{3} \phi} d s^{2}+e^{\frac{4}{3} \phi}(d z+\mathcal{A})^{2}, \\
\hat{A}_{3}(x, z) & =A_{3}(x)+A_{2}(x) \wedge d z,
\end{aligned}
$$

where $d s^{2}$ denotes the ten-dimensional metric in the string frame, and all ten-dimensional quantities are taken to be independent of the coordinate $z$ on the compactifying circle, we obtain the bosonic sector of the Type II A theory, with the Lagrangian

$$
\mathcal{L}=e e^{-2 \phi}\left(R+4(\partial \phi)^{2}-\frac{1}{12} F_{3}^{2}\right)-\frac{1}{4} e \mathcal{F}^{2}-\frac{1}{48} e F_{4}^{2}-\frac{1}{2} *\left(d A_{3} \wedge d A_{3} \wedge A_{2}\right),
$$

where $\mathcal{F}=d \mathcal{A}, F_{3}=d A_{2}$ and $F_{4}=d A_{3}-d A_{2} \wedge \mathcal{A}$. The graviton, dilaton and 2-form potential originate in the Neveu-Schwarz-Neveu-Schwarz sector of the string theory, while the 1 -form and 3 -form potentials come from the Ramond-Ramond sector.

The equations of motion for the Type IIA theory admit solutions in which the tendimensional metric $d s^{2}$ is a direct sum $d s^{2}=d s_{4}^{2}+d s_{6}^{2}$ of a four-dimensional anti de-Sitter spacetime $d s_{4}^{2}$ and a six-dimensional space $\mathcal{M}_{6}$ with metric $d s_{6}^{2}$ [19] that satisfies certain conditions given below. Substituting into the equations of motion following from (2.4), we easily see that there exist solutions with

$$
\begin{aligned}
e^{\phi} & =\lambda, \quad F_{\mu \nu \rho \sigma}=\frac{6 m}{\lambda} \epsilon_{\mu \nu \rho \sigma}, \quad \mathcal{F}_{m n}=\frac{2 \mu}{\lambda} K_{m n}, \\
R_{\mu \nu} & =-12 m^{2} g_{\mu \nu}, \quad R_{m n}=2 m^{2}\left(K_{m n}^{2}+3 g_{m n}\right), \\
m^{2} & =\mu^{2},
\end{aligned}
$$


where $m$ and $\mu$ are constants, $\lambda$ is the ten-dimensional string coupling constant, $\epsilon_{\mu \nu \rho \sigma}$ is the Levi-Civita tensor on $A d S_{4}$, and $K_{m n}$ is an harmonic 2-form on $\mathcal{M}_{6}$ with constant magnitude $K^{2}=6$. A particularly simple example of an $\mathcal{M}_{6}$ that satisfies the necessary conditions is when it is an Einstein-Kähler space of positive curvature, with $K_{m n}$ taken to be the Kähler form $J_{m n}$, in which case we see that

$$
R_{m n}=8 m^{2} g_{m n}
$$

Since the dimensional reduction from (2.1) to (2.4) is a consistent one, the solution (2.5) may be lifted back to a solution of the eleven-dimensional theory, with the metric given by (2.2):

$$
d \hat{s}^{2}=d \hat{s}_{4}^{2}+d \hat{s}_{7}^{2}
$$

where $d \hat{s}_{4}^{2}=\lambda^{-2 / 3} d s_{4}^{2}$ and

$$
d \hat{s}_{7}^{2}=\lambda^{-2 / 3} d s_{6}^{2}+\lambda^{4 / 3}(d z+\mathcal{A})^{2} .
$$

Using the fact that if $d \hat{s}_{D+1}^{2}=c^{-1} d s_{D}^{2}+c^{2}(d z+\mathcal{A})^{2}$, the vielbein components $\hat{R}_{a b}$ of the Ricci tensor of $d \hat{s}_{D+1}^{2}$ are related to the vielbein components $R_{i j}$ of $d s_{D}^{2}$ by

$$
\hat{R}_{i j}=c R_{i j}-\frac{1}{2} c^{4} \mathcal{F}_{i k} \mathcal{F}_{j}^{k}, \quad \hat{R}_{z z}=\frac{1}{4} c^{4} \mathcal{F}_{i j} \mathcal{F}^{i j}, \quad \hat{R}_{i z}=\frac{1}{2} c^{5 / 2} \nabla^{j} \mathcal{F}_{i j}
$$

we can easily see that the seven-dimensional metric $d \hat{s}_{7}^{2}$ in (2.7) is Einstein (regardless of whether or not $\mathcal{M}_{6}$ is Einstein). We find

$$
\begin{gathered}
\hat{F}_{\mu \nu \rho \sigma}=6 \hat{m} \hat{\epsilon}_{\mu \nu \rho \sigma}, \\
\hat{R}_{\mu \nu}=-12 \hat{m}^{2} \hat{g}_{\mu \nu} \\
\hat{R}_{a b}=6 \hat{m}^{2} \hat{g}_{a b},
\end{gathered}
$$

where $\hat{m}=\lambda^{1 / 3} m$ and $\hat{\epsilon}_{\mu \nu \rho \sigma}$ is the Levi-Civita tensor on $A d S_{4}$ in the rescaled metric. In fact this lifting to $D=11$ of the class of solutions (2.5) in $D=10$ gives the Freund-Rubin class of solutions [27] of eleven-dimensional supergravity, in which the metric is written as a direct sum of a four-dimensional anti de-Sitter spacetime and a seven-dimensional Einstein space $\mathcal{M}_{7}$. To be more precise, one obtains by this means all Freund-Rubin solutions where $\mathcal{M}_{7}$ can be written as a $U(1)$ bundle over some 6-manifold $\mathcal{M}_{6}$ [19], which satisfies the conditions given above. We shall refer to solutions with $m=+\mu$ and $m=-\mu$ as left-handed or right-handed respectively, since the sign dictates the orientation of $\mathcal{M}_{7}$. 
The period $\Delta z$ of of the coordinate $z$ on the compactifying circle is not arbitrary, and is determined by the harmonic form $K_{m n}$ on $\mathcal{M}_{6}$. Specifically, the period $\Delta z$ is given by

$$
\Delta z=\int \mathcal{F}
$$

or an integer fraction of this, where the integral is taken over 2 -cycles in $\mathcal{M}_{6}$. (If there is more than one 2-cycle, the periods determined by these integrals must be compatible, in order to have a well-defined solution.)

As we mentioned previously, a simple class of spaces $\mathcal{M}_{6}$ that satisfy the necessary conditions are Einstein-Kähler spaces, with $K_{m n}$ taken to be the Kähler form. One such example is provided by $C P^{3}$, endowed with the Fubini-Study metric. In this case, the seven-dimensional space $\mathcal{M}_{7}$, obtained as a $U(1)$ bundle over $C P^{3}$, is the seven-sphere. In fact the metric $d \hat{s}_{7}^{2}$ in this example is precisely the standard $S O(8)$-invariant "round" seven-sphere. We shall discuss this example in more detail in section 3 . Another example with the same topology is obtained by taking $\mathcal{M}_{6}$ to have a homogeneously "squashed" $C P^{3}$ metric, with isometry group $S O(5)$ rather than the $S U(4)$ isometry of the standard Fubini-Study metric. In this case, by choosing the squashing parameter appropriately, one obtains a solution of the type IIA theory that lifts back [19] to the squashed seven-sphere solution [29, 30] of $D=11$ supergravity. Interestingly enough, the $C P^{3}$ metric in this case is neither Einstein nor Kähler, although it is still Hermitean [19. See section 4 .

As discussed in section 5, further interesting examples of $\mathcal{M}_{7}$ spaces that are $U(1)$ fibrations can be obtained by taking $\mathcal{M}_{6}$ to be $C P^{2} \times S^{2}, S^{2} \times S^{2} \times S^{2}$, or the flag manifold $S U(3) / T^{2}$. These give rise to solutions of the Type $I I A$ theory whose liftings to $D=11$ describe compactifications where the $\mathcal{M}_{7}$ are $M^{p q r}, Q^{p q r}$ or $N^{p q r}$ spaces [19]. In the first two cases, the $\mathcal{M}_{7}$ spaces are obtained as $U(1)$ bundles characterised by their winding numbers with respect to the various Kähler structures in the $C P^{2}$ or $S^{2}$ subspaces.

Let us consider the example where $\mathcal{M}_{6}$ is $C P^{3}$, with its Fubini-Study metric, in more detail. Since the metric is Einstein-Kähler, with $R_{m n}=8 m^{2} g_{m n}$, it follows that the Ricci form is given by $\rho=8 m^{2} J$, and thus the period of the compactifying coordinate $z$ is given in terms of the first Chern class $c_{1}=\frac{1}{2 \pi} \int \rho$ by

$$
\Delta z=\frac{\pi \mu c_{1}}{2 \lambda m^{2}}
$$

Now the period $\Delta z$ is fixed once and for all, when the dimensional reduction from eleven dimensions is performed, and we may, without loss of generality, choose $\Delta z$ to have a convenient value. Let us take $\Delta z=2 \pi L$ where $\mathrm{L}$ is an arbitrary length scale. From the fact 
that the first Chern class for $C P^{3}$ is $c_{1}=4$, it then follows that $\mu=\lambda L m^{2}$. Combined with the relation $m^{2}=\mu^{2}$ in (2.5), we find that in this $C P^{3}$ solution the constant $m$ is given by

$$
m=\frac{1}{L \lambda} .
$$

As a consistency check we note that $\hat{m}=\lambda^{1 / 3} m=1 / R_{11}$, where $R_{11}=L \lambda^{2 / 3}$ is the radius of the eleventh dimension, as we would expect. This means that the R-R fields in (2.5) are actually $O\left(\lambda^{-2}\right)$

Although solutions of Type IIA supergravity, we have no conformal field theory argument to prove that the configurations (2.5) are solutions of the full string theory. There do exist conformal field theories with $A d S$ vacua [35, 36] but they involve only Neveu-Schwarz fields. However, (2.5) involves R-R field strengths which one does not know how to incorporate into conformal field theory except by expanding in powers of the R-R field, but this we cannot do because they are $O\left(\lambda^{-2}\right)$ f.

\section{The round seven-sphere}

Considered as a compactification of $D=11$ supergravity, the round $S^{7}$ yields a four dimensional $A d S$ spacetime with $N=8$ supersymmetry and $S O(8)$ gauge symmetry, for either orientation of $S^{7}$. The Kaluza-Klein mass spectrum therefore falls into $S O(8) N=8$ supermultiplets. In particular, the massless sector is described by gauged $N=8$ supergravity [15]. Since $S^{7}$ is a $U(1)$ bundle over $C P^{3}$ the same field configuration is also a solution of $D=10$ Type $I I A$ supergravity. However, the resulting vacuum has only $S U(4) \times U(1)$ symmetry and either $N=6$ or $N=0$ supersymmetry depending on the orientation of the $S^{7}$. The reason for the discrepancy is that the modes charged under the $U(1)$ are associated with the Kaluza-Klein reduction from $D=11$ to $D=10$ and are hence absent from the Type $I I A$ spectrum originating from the massless Type $I I A$ supergravity. In other words, they are Dirichlet 0-branes and hence absent from the perturbative string spectrum. There is thus more non-perturbative gauge symmetry and supersymmetry than perturbative. The right-handed orientation is especially interesting because the perturbative theory has no supersymmetry at all! See Table 1 (where we are using the notation of 28] for $S U(4)$ representations). It is interesting to note that the $D=4$ massless states in the left-handed vacuum originate from the $n=0$ massless level and $n=1,2$ massive levels in $D=10$, whereas in the right-handed vacuum they originate from $n=0,1,2,3,4$ levels.

\footnotetext{
${ }^{4}$ We are grateful to E. Witten for correspondence on this point.
} 


\begin{tabular}{|c|c|l|l|}
\hline Spin & $S O(8)$ reps & Left $S U(4) \times U(1)$ reps & Right $S U(4) \times U(1)$ reps \\
\hline \hline 2 & 1 & $1_{0}$ & $1_{0}$ \\
$\frac{3}{2}$ & $8_{s}$ & $6_{0}+1_{2}+1_{-2}$ & $4_{1}+\overline{4}_{-1}$ \\
1 & 28 & $1_{0}+15_{0}+6_{2}+6_{-2}$ & $1_{0}+15_{0}+6_{2}+6_{-2}$ \\
$\frac{1}{2}$ & $56_{s}$ & $6_{0}+10_{0}+\overline{10}_{0}+15_{2}+15_{-2}$ & $4_{1}+\overline{4}_{-1}+20_{1}+\overline{0_{-1}}+4_{-3}+\overline{4}_{3}$ \\
$0^{+}$ & $35_{v}$ & $15_{0}+10_{-2}+\overline{10}_{2}$ & $15_{0}+10_{-2}+\overline{10}_{2}$ \\
$0^{-}$ & $35_{c}$ & $15_{0}+10_{2}+\overline{10}_{-2}$ & $1_{0}+20^{\prime}+6_{2}+6_{-2}+1_{4}+1_{-4}$ \\
\hline
\end{tabular}

Table 1: The massless multiplet under $S O(8) \rightarrow S U(4) \times U(1)$

Similar remarks apply to the massive spectrum. By way of examples, we discuss the decomposition of the first two massive levels, for both the left-handed $N=6$ vacuum in Appendix A, and the right-handed $N=0$ vacuum in Appendix B.

\section{The squashed seven-sphere}

The squashed $S^{7}$ solution of $D=11$ supergravity yields an $M$-theory vacuum with $S O(5) \times$ $S U(2)$ and $N=1$ or $N=0$ for either left or right orientation [29, 30]. Again it corresponds to a $U(1)$ bundle over a squashed $C P^{3}$ and may also be interpreted [19] as a solution of Type $I I A$ supergravity but with only $S O(5) \times U(1)$. Since the single massless gravitino of the left-squashed solution is a singlet under $U(1)$, the perturbative vacua have the same supersymmetry as the non-perturbative.

\section{$5 \quad M(3,2)$}

The $M^{p q r}$ spaces can be described very simply as $U(1)$ bundles over $C P^{2} \times S^{2}$. The most natural way to classify them is in the $M(m, n)$ notation of [31], where $m$ and $n$ are the winding numbers of the $U(1)$ fibres over $C P^{2}$ and $S^{2}$ respectively. In the notation of [33], the space $M^{p q r}$ corresponds to $M(m, n)$ with $p=m / r$ and $q=n / r$, where $r$ is the greatest common divisor of $m$ and $n$. For each pair of integers $m$ and $n$, there is an Einstein metric on the 7-dimensional bundle space, where the scale sizes of the standard metrics on $C P^{2}$ and $S^{2}$ are chosen appropriately [31]. In the case $M(3,2)$, the space admits two Killing spinors; when $2 m \neq 3 n$, there are no Killing spinors.

This example is particularly interesting for two reasons. First, it is a special case of 
the $M^{p q r}$ spaces constructed by Witten [32] displaying $S U(3) \times S U(2) \times U(1)$ symmetry. This symmetry is the same even when considered as a solution of Type $I I A$ supergravity. Secondly, as an $M$-theory vacuum the right-handed orientation has $N=0$ [15] and the left-handed orientation has $N=2$ [33], but since both gravitinos are charged under the $U(1)$, the perturbative theory has no supersymmetry. It fact, the perturbative theory is especially bizarre: it has no fermions whatsoever! This because $M^{p q r}$ is a $U(1)$ bundle over $C P^{2} \times S^{2}$ and although it has a spin structure, $C P^{2} \times S^{2}$ does not. Only spinors with appropriate charges under the R-R 2-form field strength on $C P^{2}$ can be defined. These are $\operatorname{spin}^{c}$ spinors or generalized spinors [34]. Hence the perturbative spectrum of such a Type II $A$ vacuum is entirely bosonic: all the fermions are Dirichlet 0-branes!

\section{Perturbative versus non-perturbative}

The states that are charged under the R-R $U(1)$ are Dirichlet 0-branes whose masses are of order $1 / \lambda$ and which therefore become very heavy for weak coupling of the $D=10$ Type II $A$ string. On the other hand they belong to the same supermultiplet as the neutral states and should therefore be degenerate in mass with them (in the sense of $A d S$ supersymmetry). Consistency therefore demands that all the four-dimensional massive states have masses of order $1 / \lambda$, whether Dirichlet 0-branes or not, and that the entire massive Kaluza-Klein spectrum is, in this sense, non-perturbative. This is indeed the case because the massive spectrum associated with the compactification from $D=10$ to $D=4$ on $C P^{3}$ is governed by the parameter $m$ which, from (2.13) does indeed go like $1 / \lambda$. Note, however, that the massless sector remains massless for any value of $\lambda$ and so it still makes sense to talk about "perturbative" supersymmetry whose gravitinos are neutral under the $U(1)$ and the extra "non-perturbative" supersymmetry whose gravitinos are Dirichlet 0-branes. Similar remarks apply to the gauge symmetry and the massless gauge bosons.

The $A d S$ vacua of Type $I I A$ supergravity considered in this paper have spacetime cosmological constant $m^{2} \sim 1 / \lambda^{2}$ and are, of course, very different from the Minkowski vacua. Note also that the volume $V$ of $\mathcal{M}_{6}$ scales like $m^{-6} \sim \lambda^{6}$. This has a strange consequence. From the four dimensional point of view, the string coupling $\lambda_{4}$ is related to the ten-dimensional string coupling $\lambda$ appearing in (2.4) by

$$
\frac{1}{\lambda_{4}^{2}}=\frac{V}{L^{6} \lambda^{2}}
$$

In Minkowski space string theory, the volume $V$ is given by the vev of a massless modulus field and its value is independent of the string coupling which is determined by the vev 
of the dilaton $\phi$. Consequently, weak coupling in $D=10$ means weak coupling in $D=$ 4. In $A d S$, however, the volume field belongs to the massive sector 115. In the case of the $C P^{3}$ for example, it is the singlet scalar in the second massive level that comes from the singlet in the round $S^{7}$ supermultiplet with spins $\left\{2, \frac{3}{2}, 1, \frac{1}{2}, 0^{+}, 0^{-}\right\}$transforming as $\left\{35_{v}, 56_{s}+224_{v s}, 28+350+567_{v}, 8_{s}+160_{s}+672_{v c}+840_{s}, 1+294_{v}+300,35_{s}+840_{s}^{\prime}\right\}$, as discussed in Appendices A and B. Moreover, the vev of this scalar is not a free parameter but is itself fixed by the string coupling. From (2.5) and (2.13), we see that

$$
V \sim m^{-6}=L^{6} \lambda^{6}
$$

Thus we reach the bizarre conclusion that

$$
\lambda_{4} \sim \frac{1}{\lambda^{2}}
$$

and the four-dimensional string coupling grows as the ten-dimensional string coupling shrinks! Similar remarks apply to the non-abelian gauge coupling constant $e(S O(8)$ in the case of the round $S^{7}$ ). Since one of the gauge bosons is just the $U(1)$ gauge field appearing in (2.4), we have

$$
e^{2}=\frac{L^{6}}{V} \sim \frac{1}{\lambda^{6}}
$$

\section{Conclusions}

We have seen how supersymmetry can appear very differently when viewed from the perturbative Type $I I A$ string perspective (vacua corresponding to solutions of massless Type $I I A$ supergravity whose spectrum is neutral under the R-R charge) and the non-perturbative $M$-theory perspective (vacua corresponding to solutions of $D=11$ supergravity whose spectrum includes Dirichlet 0-branes). A summary of perturbative versus non-perturbative symmetries is given in Table 2. In particular, the non-perturbative vacuum may have unbroken supersymmetry even when the perturbative vacuum has none.

We cannot resist asking whether this could be a model of the real world in which you can have your supersymmetry and eat it too 5 . The problem with such a scenario, of course, is that God does not do perturbation theory and presumably an experimentalist would measure God's real world and not what a perturbative string theorist thinks is the real

\footnotetext{
${ }^{5} \mathrm{~A}$ scheme in which you can have all the benefits of unbroken supersymmetry while appearing to inhabit a non-supersymmetric world has also been proposed by Witten [37] but his mechanism is very different from ours. In particular, our vacua necessarily have non-vanishing cosmological constant unless cancelled by fermion condensates 38 .
} 


\begin{tabular}{|c|cc|cc|}
\hline Compactification & \multicolumn{2}{|r|}{ Perturbative Type IIA } & & Nonperturbative M-theory \\
\hline \hline Left round $S^{7}$ & $N=6$ & $S U(4) \times U(1)$ & $N=8$ & $S O(8)$ \\
Right round $S^{7}$ & $N=0$ & $S U(4) \times U(1)$ & $N=8$ & $S O(8)$ \\
Left squashed $S^{7}$ & $N=1$ & $S O(5) \times U(1)$ & $N=1$ & $S O(5) \times S U(2)$ \\
Right squashed $S^{7}$ & $N=0$ & $S O(5) \times U(1)$ & $N=0$ & $S O(5) \times S U(2)$ \\
Left $M(3,2)$ & $N=0$ & $S U(3) \times S U(2) \times U(1)$ & $N=2$ & $S U(3) \times S U(2) \times U(1)$ \\
Right $M(3,2)$ & $N=0$ & $S U(3) \times S U(2) \times U(1)$ & $N=0$ & $S U(3) \times S U(2) \times U(1)$ \\
\hline
\end{tabular}

Table 2: Perturbative versus non-perturbative symmetries

world. Unless, for some unknown reason, the experimentalist's apparatus is so primitive as to be unable to detect Ramond-Ramond charge in which case he or she would conclude that the world has no unbroken supersymmetry.

\section{Acknowledgements}

We have enjoyed useful conversations with Karim Benakli, Jacques Distler, Glennys Farrar, Hermann Verlinde and Edward Witten.

\section{A The first two massive levels on $S^{7}: N=6$ decomposition}

Let us consider the first massive level [15] on $S^{7}$. Under the $N=8 \rightarrow N=6, S O(8) \rightarrow$ $S U(4) \times U(1)$ decomposition of the left-handed $S^{7}$, it yields the decomposition given in Table 3. Note that there are no $U(1)$ singlets so all these states are Dirichlet 0-branes, absent from the Type $I I A$ Kaluza-Klein spectrum in going from $D=10$ to $D=4$. This is a general feature of the odd numbered levels.

Next we look at the second massive level in Table 1 . Since the numbers of representations in the decompositions rapidly become large, we shall just present the subset of states that carry no Ramond-Ramond charges and which survive in the truncation to the Type IIA spectrum forming $N=6$ supermultiplets. 


\begin{tabular}{|c|c|c|}
\hline Spin & $S O(8)$ reps & Left $S U(4) \times U(1)$ reps \\
\hline 2 & $8 v$ & $4_{-1}+\overline{4}_{1}$ \\
\hline \multirow[t]{2}{*}{$\overline{2}$} & $8_{c}$ & $4_{1}+\overline{4}_{-1}$ \\
\hline & $56_{c}$ & $4_{1}+\overline{4}_{-1}+20_{1}+\overline{20}_{1}+4_{-3}+\overline{4}_{3}$ \\
\hline \multirow[t]{2}{*}{1} & $56_{v}$ & $4_{-1}+\overline{4}_{1}+20_{-1}+\overline{20}_{1}+4_{3}+\overline{4}_{-3}$ \\
\hline & $160_{v}$ & $4_{-1}+\overline{4}_{1}+20_{-1}+\overline{20}_{1}+20_{3}+\overline{20}_{-3}+36_{-1}+3 \overline{6}_{1}$ \\
\hline \multirow[t]{2}{*}{$\frac{1}{2}$} & $160_{c}$ & $4_{1}+\overline{4}_{-1}+20_{1}+2 \overline{0}_{-1}+20_{-3}+\overline{20}_{3}+36_{1}+3 \overline{36}_{-1}$ \\
\hline & $224_{v c}$ & $20_{1}+\overline{20}_{-1}+20_{1}^{\prime \prime}+\overline{20}_{-1}^{\prime \prime}+36_{1}+\overline{36}_{-1}+36_{-3}+\overline{36}_{3}$ \\
\hline $0^{+}$ & $112_{v}$ & $20_{3}^{\prime \prime}+\overline{20}_{-3}^{\prime \prime}+36_{-1}+\overline{36}_{1}$ \\
\hline $0^{-}$ & $224_{c v}$ & $20_{-1}+\overline{20}_{1}+20_{-1}^{\prime \prime}+\overline{20}_{1}^{\prime \prime}+36_{-1}+\overline{36}_{1}+36_{3}+\overline{36}_{-3}$ \\
\hline
\end{tabular}

Table 3: The first massive level under $S O(8) \rightarrow S U(4) \times U(1)$

\begin{tabular}{|c|c|l|}
\hline Spin & $S O(8)$ reps & Left $S U(4) \times U(1)$ reps \\
\hline \hline 2 & $35_{v}$ & $15_{0}+\cdots$ \\
$\frac{3}{2}$ & $56_{s}$ & $6_{0}+10_{0}+\overline{10}_{0}+\cdots$ \\
& $224_{v s}$ & $10_{0}+\overline{10}_{0}+64_{0}+\cdots$ \\
1 & 28 & $1_{0}+15_{0}+\cdots$ \\
& 350 & $15_{0}+15_{0}+20_{0}^{\prime}+45_{0}+\overline{45}+\cdots$ \\
& $567_{v}$ & $15_{0}+45_{0}+\overline{45} \overline{5}_{0}+84_{0}+\cdots$ \\
$\frac{1}{2}$ & $8_{s}$ & $6_{0}+\cdots$ \\
& $160_{s}$ & $6_{0}+6_{0}+64_{0}+\cdots$ \\
& $672_{v c}$ & $64_{0}+70_{0}+7 \overline{0}_{0}+\cdots$ \\
& $840_{s}$ & $6_{0}+10_{0}+\overline{10} \overline{0}_{0}+64_{0}+64_{0}+70_{0}+7 \overline{0}_{0}+\cdots$ \\
$0^{+}$ & 1 & $1_{0}$ \\
& $294_{v}$ & $0+\cdots$ \\
& 300 & $1_{0}+15_{0}+20_{0}^{\prime}+84_{0}+\cdots$ \\
$0^{-}$ & $35_{s}$ & $1_{0}+20_{0}^{\prime}+\cdots$ \\
& $840_{s}^{\prime}$ & $20_{0}^{\prime}+35_{0}+\overline{35} \overline{5}_{0}+45_{0}+\overline{45_{0}}+84_{0}+\cdots$ \\
\hline
\end{tabular}

Table 4: The second massive level under $S O(8) \rightarrow S U(4) \times U(1)$ 


\section{B The first two massive levels on $S^{7}: N=0$ decomposition}

Again let us first consider the first massive level on $S^{7}$. Under the $N=8 \rightarrow N=0$, $S O(8) \rightarrow S U(4) \times U(1)$ decomposition of the right-handed $S^{7}$, it yields the decomposition given in Table 5. Note that the surviving singlets are all fermions. This is a general feature of the odd numbered levels.

Next we look at the second massive level in Table 6. Note that the surviving singlets are all bosons. This is a general feature of all even numbered levels. Keeping just the $U(1)$ singlets thus yields a massive spectrum of bosons and fermions but which do not form supermultiplets. The supermultiplet structure of the spectrum becomes apparent only when the Dirichlet 0-branes are incorporated.

The coupling constants appearing in the truncated tree-level Lagrangian would take on their supersymmetric values but this would not persist at the quantum level unless the Dirichlet 0-branes were re-introduced. 


\begin{tabular}{|c|c|c|}
\hline Spin & $S O(8)$ reps & Right $S U(4) \times U(1)$ reps \\
\hline 2 & $8 v$ & $4_{-1}+\overline{4}_{1}$ \\
\hline \multirow[t]{2}{*}{$\frac{3}{2}$} & $8 c$ & $1_{2}+1_{-2}+6_{0}$ \\
\hline & $56_{c}$ & $6_{0}+10_{0}+\overline{10}_{0}+15_{2}+15_{-2}$ \\
\hline \multirow[t]{2}{*}{1} & $56_{v}$ & $4_{-1}+\overline{4}_{1}+20_{-1}+\overline{20}_{1}+4_{3}+\overline{4}_{-3}$ \\
\hline & $160_{v}$ & $4_{-1}+\overline{4}_{1}+20_{-1}+\overline{20}_{1}+20_{3}+\overline{20}_{-3}+36_{-1}+\overline{36}_{1}$ \\
\hline \multirow[t]{2}{*}{$\frac{1}{2}$} & $160_{c}$ & $1_{2}+1_{-2}+6_{0}+6_{0}+6_{4}+6_{-4}+15_{2}+15_{-2}$ \\
\hline & $224_{v c}$ & $10_{0}+\overline{10}_{0}+64_{0}+15_{2}+15_{-2}+45_{-2}+\overline{45}_{2}+10_{-4}+\overline{10}_{4}$ \\
\hline $0^{+}$ & $112_{v}$ & $20_{3}^{\prime \prime}+\overline{20}_{-3}^{\prime \prime}+36_{-1}+\overline{36}_{1}$ \\
\hline $0^{-}$ & $224_{c v}$ & $4_{-1}+\overline{4}_{1}+4_{3}+\overline{4}_{-3}+4_{-5}+\overline{4}_{5}+20_{-1}+\overline{20}_{1}+20_{3}+\overline{20}_{-3}+60_{-1}+\overline{60}_{1}$ \\
\hline
\end{tabular}

Table 5: The first massive level under $S O(8) \rightarrow S U(4) \times U(1)$

\begin{tabular}{|c|c|l|}
\hline Spin & $S O(8)$ reps & Right $S U(4) \times U(1)$ reps \\
\hline \hline 2 & $35_{v}$ & $15_{0}+\cdots$ \\
$\frac{3}{2}$ & $56_{s}$ & $0+\cdots$ \\
& $224_{v s}$ & $0+\cdots$ \\
1 & 28 & $1_{0}+15_{0}+\cdots$ \\
& 350 & $15_{0}+15_{0}+20_{0}^{\prime}+45_{0}+\overline{45} 5_{0}+\cdots$ \\
& $567_{v}$ & $15_{0}+45_{0}+\overline{45} 5_{0}+84_{0}+\cdots$ \\
$\frac{1}{2}$ & $8_{s}$ & $0+\cdots$ \\
& $160_{s}$ & $0+\cdots$ \\
& $672_{v c}$ & $0+\cdots$ \\
& $840_{s}$ & $0+\cdots$ \\
$0^{+}$ & 1 & $1_{0}$ \\
& $294_{v}$ & $0+\cdots$ \\
& 300 & $1_{0}+15_{0}+20_{0}^{\prime}+84_{0}+\cdots$ \\
$0^{-}$ & $35_{s}$ & $15_{0}+\cdots$ \\
& $840_{s}^{\prime}$ & $10_{0}+45_{0}+\overline{4} 5_{0}+175_{0}$ \\
\hline
\end{tabular}

Table 6: The second massive level under $S O(8) \rightarrow S U(4) \times U(1)$ 


\section{References}

[1] M. J. Duff, P. Howe, T. Inami and K. S. Stelle, Superstrings in $D=10$ from supermembranes in $D=11$, Phys. Lett. B191 (1987) 70.

[2] M. J. Duff and J. X. Lu, Duality rotations in membrane theory, Nucl.Phys. B347 (1990) 394.

[3] C. M. Hull and P. K. Townsend, Unity of superstring dualities, Nucl. Phys. B438 (1995) 109.

[4] P. K. Townsend, The eleven-dimensional supermembrane revisited, Phys.Lett.B350 (1995) 184.

[5] E. Witten, String theory dynamics in various dimensions, Nucl. Phys. B443 (1995) 85.

[6] M. J. Duff, J. T. Liu and R. Minasian, Eleven-dimensional origin of string/string duality: A one loop test, Nucl.Phys. B452 (1995) 261.

[7] K. Becker, M. Becker and A. Strominger, Fivebranes, membranes and nonperturbative string theory, Nucl.Phys. B456 (1995) 130.

[8] J. H. Schwarz, The power of M-theory, Phys. Lett. B360 (1995) 13.

[9] P. Horava and E. Witten, Heterotic and Type I string dynamics from eleven dimensions, Nucl. Phys. B460 (1996) 506.

[10] P. Townsend, D-Branes From M-Branes, Phys. Lett. B373 (1996) 68.

[11] O. Aharony, J. Sonnenschein and S. Yankielowicz, Interactions of strings and D-branes from M-theory, Nucl. Phys. B474 (1996) 309.

[12] M. J. Duff, M-theory (the theory formerly known as strings), I. J. M. P A11 (1996) 5623.

[13] T. Banks, W. Fischler, S. H. Shenker and L. Susskind, $M$ theory as a matrix model: a conjecture, Phys. Rev. D55 (1997) 5112.

[14] J. Polchinski, Dirichlet-branes and Ramond-Ramond charges, Phys. Rev. Lett. 75 (1995) 4724. 
[15] M. J. Duff, B. E. W. Nilsson and C. N. Pope, Kaluza-Klein supergravity, Phys. Rep. 130 (1986) 1.

[16] E. Cremmer, B. Julia and J. Scherk, Supergravity theory in 11 dimensions, Phys. Lett. B76 (1978) 409.

[17] M. J. Duff, B. E. W. Nilsson and C. N. Pope, Compactification of $D=11$ supergravity on $K 3 \times T^{3}$, Phys. Lett. B129 (1983) 39 .

[18] A. Lucas, B. A. Ovrut and D. Waldram, Stabilizing dilaton and moduli vacua in string and $M$-theory, hep-th/9611204.

[19] B. E. W. Nilsson and C. N. Pope, Hopf fibration of eleven-dimensional supergravity, Class. Quantum Grav. 1 (1984) 499.

[20] M. J. Duff and K. Stelle, Multimembrane solutions of $d=11$ supergravity, Phys.Lett. B253 (1991) 113.

[21] G. W. Gibbons and P. K. Townsend, Vacuum interpolation in supergravity via super p-branes, Phys. Rev. Lett. 71 (1993) 3754.

[22] M. J. Duff, G. W. Gibbons and P. K. Townsend, Macroscopic superstrings as interpolating solitons, Phys. Lett. B332 (1994) 321.

[23] M. B. Green and J. S. Schwarz, Covariant description of superstrings, Phys. Lett. B136 (1984) 367.

[24] E. Bergshoeff, E. Sezgin and P. Townsend, Supermembranes and eleven-dimensional supergravity Phys.Lett. B209 (1988) 451.

[25] I. G. Campbell and P. C. West, $N=2 D=10$ nonchiral supergravity and its spontaneous compactification, Nucl. Phys.B243 (1984) 112.

[26] M. Huq and M. A. Namazie, Kaluza-Klein supergravity in ten dimensions, Class. Quant. Grav. 2 (1985) 293.

[27] P. G. O. Freund and M. A. Rubin, Dynamics of dimensional reduction, Phys. Lett. B97 (1980) 233.

[28] R. Slansky, Group theory for unified model building, Phys. Rep. 79 (1981) 1. 
[29] M. A. Awada, M. J. Duff and C. N. Pope, $N=8$ Supergravity Breaks Down to $N=1$, Phys. Rev. Lett. 50, 294 (1983).

[30] M. J. Duff, B. E. W. Nilsson and C. N. Pope, Spontaneous Supersymmetry Breaking by the Squashed Seven-Sphere, Phys. Rev. Lett. 50, 2043 (1983).

[31] D.N. Page and C. N. Pope, Stability analysis of compactifications of $D=11$ supergravity with $S U(3) \times S U(2) \times U(1)$ symmetry, Phys. Lett. B145 (1984) 337 .

[32] E. Witten, Search for a realistic Kaluza Klein theory, Nucl. Phys. B186 (1981) 412.

[33] L. Castellani, R. D'Auria and P. Fre, $S U(3) \times S U(2) \times U(1)$ from $D=11$ supergravity, Nucl. Phys. B239 (1984) 610.

[34] S. W. Hawking and C. N. Pope, Generalized spin structures in quantum gravity, Phys. Lett. B73 (1978) 42.

[35] I. Antoniadis, C. Bachas and A. Sagnotti, Gauged supergravity vacua in string theory, Phys. Lett. B235 (1990) 255.

[36] I. Antoniadis, S. Ferrara and C. Kounnas, Exact supersymmetric string solutions in curved gravitational backgrounds, Nucl. Phys. B421 (1994) 343.

[37] E. Witten, Strong coupling and the cosmological constant, Mod. Phys. Lett. A10 (1995) 2153.

[38] M. J. Duff and C. Orzalesi, The cosmological constant in spontaneously compactified $D=11$ supergravity, Phys. Lett. B122 (1983) 37. 